\title{
VARIAÇÃO DA EVAPOTRANSPIRAÇÃO DE REFERÊNCIA CALCULADA EM DIFERENTES INTERVALOS DE TEMPO
}

\begin{abstract}
ÁLVARO J. BACK ${ }^{1}$
RESUMO: Valores de evapotranspiração de referência foram calculados em intervalos de um, cinco, dez, quinze dias e mensal. Foram utilizados dados meteorológicos da Estação Meteorológica de Urussanga - SC (latitude $28^{\circ} 31^{\prime}$ S, longitude $49^{\circ} 19^{\prime} \mathrm{W}$ ) referentes ao período de 1981 a 2004 . A evapotranspiração de referência foi calculada pelo método de Penman-Monteith com base nas médias das variáveis meteorológicas dos diferentes períodos estudados. Observou-se que não houve diferenças significativas nas médias de evapotranspiração calculadas nos diversos intervalos de tempo. No entanto, houve diferenças significativas na dispersão dos valores em torno da média. Para valores diários, foi obtido coeficiente de variação (C.V.) entre 30 e 40\%, e para valores mensais, variou entre $6 \%$ e $12 \%$. Observou-se, também, que os dados de evapotranspiração se ajustaram à distribuição Beta, e assim foram estimados, para cada mês, os valores de evapotranspiração de referência com probabilidades de $5 ; 10 ; 25 ; 50 ; 75 ; 90$ e $95 \%$.
\end{abstract}

PALAVRAS-CHAVE: balanço hídrico, probabilidade, evapotranspiração.

\section{VARIATION OF THE REFERENCE EVAPOTRANSPIRATION CALCULATED FOR DIFFERENT TIMES INTERVAL}

\begin{abstract}
Reference evapotranspiration values were calculated for time interval of one, five, ten, fifteen days and monthly. Meteorological series data from 1981 to 2004 from Urussanga Weather Station (latitude $28^{\circ} 31^{\prime} \mathrm{S}$, longitude $49^{\circ} 19^{\prime} \mathrm{W}$ ) were used to calculate reference evapotranspiration with Penman-Monteith method with mean of meteorological variables for every time intervals. No difference was observed in average reference evapotranspiration computed in all time steps. However, some difference in variations of data around the average was observed. For daily value, the variation coefficient varies from 30 to $40 \%$ and for monthly values de coefficient variation varied from 6 to $12 \%$. It was observed that the reference evapotranspirations adjusted very well to Beta distribution probability, and the reference evapotranspiration with probability of $5 ; 10 ; 25 ; 50 ; 75 ; 90$ e $95 \%$ was estimated.
\end{abstract}

KEYWORDS: water budget, probability, evapotranspiration.

\section{INTRODUÇÃO}

O conhecimento da evapotranspiração é de grande importância na estimativa da necessidade de irrigação das culturas, bem como para o manejo racional de recursos hídricos. Vários modelos de balanço hídrico são empregados com a finalidade de dimensionar sistemas de irrigação, manejar projetos de irrigação ou estudar probabilidades de ocorrências de estiagens ou excessos hídricos. Nesses modelos, utilizam-se, em geral, como entradas de água no sistema, a precipitação e a irrigação, e como principal saída, a evapotranspiração das culturas. Dados de precipitação medida podem ser obtidos com relativa facilidade, no entanto existe carência de informações sobre evapotranspiração das culturas. Devido às dificuldades de medida da evapotranspiração das culturas, essa é estimada com base nos valores da evapotranspiração de referência (ETo) e no coeficiente de cultura.

Existem diversos métodos de estimativa da evapotranspiração de referência e alguns somente podem ser aplicados para estimativas mensais, outros para intervalos decendiais ou maiores

\footnotetext{
${ }^{1}$ Eng ${ }^{\circ}$ Agrônomo, Pesquisador, Epagri, Estação Experimental de Urussanga, Rodovia SC 446, km 19, Caixa Postal 049, Urussanga - SC, ajb@epagri.sc.gov.br

Recebido pelo Conselho Editorial em: 23-6-2005
}

Aprovado pelo Conselho Editorial em: 12-12-2006 
(LIMA, 1991). De acordo com SMITH et al. (1991), o método de Penman-Monteith foi unanimemente aceito por pesquisadores de diversos países, como aquele de melhor desempenho para a estimativa de ETo. Esse método tem sido recomendado para intervalos diários, e o de ALLEN et al. (1998) apresenta a metodologia para cálculo em intervalos horários.

A escolha do intervalo de tempo para o cálculo da ETo depende do objetivo e da precisão desejada e do intervalo de tempo usado no cálculo das médias da variável meteorológica. JENSEN et al. (1990) relatam que a equação de Penman-Monteith é mais precisa quando usada na base horária e somando seus valores para obter a estimativa diária. Segundo os autores, exemplos de cálculos mostram claramente que, quando usados os elementos climáticos diários, a equação de Penman-Monteith pode proporcionar estimativas precisas de ETo.

DOOREMBOS \& PRUITT (1977) advertem que o uso de dados climáticos médios em equações combinadas pode conduzir a erros significativos, resultantes das combinações entre os elementos climáticos. Segundo ALLEN et al. (1998), apesar da não-linearidade entre a equação de Penman-Monteith e alguns parâmetros meteorológicos, médias de dez dias ou mensais dos dados meteorológicos podem ser usadas para calcular a evapotranspiração média de períodos de dez dias ou mensais. Ainda segundo esses autores, o valor da evapotranspiração média mensal calculada com a média mensal dos dados meteorológicos é muito similar à média da evapotranspiração diária calculada com os dados diários para aquele mês.

Neste estudo, avaliou-se a variação dos valores de evapotranspiração de referência calculada para intervalos de um, cinco, dez, quinze dias ou mensais.

\section{MATERIAL E MÉTODOS}

Foram utilizados os dados diários de temperatura máxima e mínima do ar, umidade relativa do ar, velocidade do vento e insolação registradas na estação meteorológica de Urussanga - SC (latitude 28³1'S, longitude 49¹9’W) no período de 1981 a 2004.

Foram calculadas as médias das variáveis meteorológicas para intervalos de um dia, cinco dias (pentadas), dez dias (decêndios), quinze dias (quinzenas) e mensais. Nos meses com 31 dias, o último período do mês foi calculado com um dia a mais e, em fevereiro, o último período foi calculado com um ou dois dias a menos, conforme se o ano era bissexto ou não. Com as médias das variáveis meteorológicas para cada período considerado, calculou-se a evapotranspiração de referência pelo método e Penman-Monteith, de acordo com a equação:

$$
\text { ETo }=\frac{0,408 \Delta(\mathrm{Rn}-\mathrm{G})+\gamma \frac{900}{(\mathrm{~T}+273)} \mathrm{U}_{2}(\mathrm{es}-\mathrm{ea})}{\Delta+\gamma(1+0,34 \mathrm{U} 2)}
$$

em que,

ETo - evapotranspiração de referência, $\mathrm{mm} \mathrm{d}^{-1}$;

$\mathrm{R}_{\mathrm{n}}$ - saldo de radiação à superfície, $M J \mathrm{~m}^{-2} \mathrm{~d}^{-1}$;

$\mathrm{G}$ - fluxo de calor sensível no solo, $\mathrm{MJ} \mathrm{m}^{-2} \mathrm{~d}^{-1}$;

$\mathrm{T}$ - temperatura média do ar a $2 \mathrm{~m}$ de altura, ${ }^{\circ} \mathrm{C}$;

$\mathrm{U}_{2}$ - velocidade do vento a $2 \mathrm{~m}$ de altura, $\mathrm{m} \mathrm{s}^{-1}$;

es - pressão de saturação de vapor, $\mathrm{kPa}$;

ea - pressão atual de vapor, $\mathrm{kPa}$;

$\Delta$ - declividade da curva de pressão de saturação, $\mathrm{kPa}^{\circ} \mathrm{C}^{-1}$, e

$\gamma$ - constante psicrométrica, $\mathrm{kPa}{ }^{\circ} \mathrm{C}^{-1}$.

Os cálculos foram realizados conforme descrito por ALLEN et al. (1998), sendo a temperatura média diária estimada como a média entre a temperatura máxima e a mínima no dia. A radiação solar foi estimada com o uso da equação de Angstron, considerando os valores de $\mathrm{a}=$ $0,25 \mathrm{e} \mathrm{b}=0,50$. A irradiância solar que atinge o topo da atmosfera foi calculada para o dia compreendido no meio do intervalo $\left(3^{\mathrm{o}} ; 5^{\mathrm{o}} ; 8^{\mathrm{O}}\right.$ e $15^{\mathrm{o}}$ dia para pêntadas, decêndios, quinzenas e 
mensal, respectivamente). $\mathrm{O}$ albedo foi considerado como 0,23. Para todos os intervalos, o fluxo de calor no solo foi desprezado $(\mathrm{G}=0)$.

Para verificar a freqüência dos valores de evapotranspiração, foram calculados os valores esperados de evapotranspiração de referência com probabilidades de $5 ; 10 ; 25 ; 50 ; 75 ; 90$ e $95 \%$, utilizando a distribuição normal e a distribuição Beta, com a seguinte função densidade de probabilidade:

$$
f(x)=\frac{\Gamma(\alpha+\beta)}{\Gamma(\alpha) \Gamma(\beta)} x^{\alpha-1}(1-x)^{\beta-1}
$$

sendo $0 \leq \mathrm{x} \leq 1 ; \alpha>0 ; \beta>0$.

O ajuste dos parâmetros da distribuição Beta e os cálculos dos valores esperados foram realizados conforme descrito por ASSIS et al. (1996). Para verificar o ajuste das distribuições de probabilidade aos dados de evapotranspiração de referência, foi realizado o teste de KolmogorovSmirnov, a 5\% de significância, conforme descrito por KITE (1978).

\section{RESULTADOS E DISCUSSÃO}

$\mathrm{Na}$ Tabela 1, encontram-se as medidas de tendência central, de dispersão e assimetria para cada mês dos valores de evapotranspiração de referência calculada para diferentes intervalos de tempo. Observam-se diferenças inferiores a 5\% nos valores médios de evapotranspiração calculadas nos diversos períodos, dentro de cada mês. Por meio da análise de variância, não foram observadas diferenças significativas $(\mathrm{p}>0,10)$ entre essas médias de evapotranspiração de referência, calculadas dentro de cada mês. Isso confirma as observações de ALLEN et al. (1998), de que, embora não haja linearidade entre as variáveis meteorológicas envolvidas e a equação de Penman-Monteith, não se observam diferenças nas médias mensais da evapotranspiração de referência calculada, com a média dos valores em períodos de dez dias, ou mensais com as médias calculadas dos valores diários de evapotranspiração.

No entanto, para os menores intervalos, observa-se maior dispersão dos valores em torno da média, com valores de coeficiente de variação entre 30 e $40 \%$ para os cálculos com dados diários. Dessa observação, pode-se inferir que a utilização de valores médios diários de evapotranspiração de referência em modelos de balanço hídrico seriado pode implicar erros na estimativa do consumo de água. SAAD \& SCALOPPI (1988) já alertavam que a grande variabilidade dos valores assumidos pelos parâmetros meteorológicos durante o período de máxima exigência hídrica das culturas acarreta considerável dispersão dos valores calculados da evapotranspiração, sugerindo análise da distribuição de freqüência dos valores estimados para fins de dimensionamento de sistemas de irrigação. Para os períodos maiores, essa variação diminui, e no intervalo mensal, o coeficiente de variação ficou entre 6 e $12 \%$.

Os valores do coeficiente de assimetria (As) para todos os períodos se apresentaram próximos de zero, sendo, na maioria dos períodos, valores positivos. Os valores do coeficiente de assimetria próximo a zero é indicação de que esses dados têm distribuição aproximadamente normal. Em junho e julho, foram encontrados valores de coeficiente de assimetria acima de 1,0. Esses valores se devem, em parte, à presença de alguns valores extremos de evapotranspiração de referência, calculada devido a alguns dados relativamente altos de velocidade do vento registrados.

Vários trabalhos indicam a distribuição normal (ARRUDA \& BARROSO, 1984; SAAD \& SCALOPPI, 1988) e a distribuição Beta (SAAD, 1990) para o estudo da frequiência da evapotranspiração de referência. Na Tabela 2, encontram-se os valores dos desvios máximos entre os valores observados e os valores estimados de evapotranspiração de referência para a distribuição normal e a distribuição Beta. Observa-se que os menores desvios são obtidos com a distribuição Beta e, para o intervalo diário em todos os meses, com exceção de maio, a distribuição normal é rejeitada pelo teste de Kolmogorov-Smirnov. Dessa forma, foi utilizada a distribuição Beta na estimativa dos valores de evapotranspiração estimada com diferentes probabilidades. 
TABELA 1. Medidas de tendência central, dispersão e assimetria da evapotranspiração de referência, calculada em diferentes intervalos de tempo com os dados de Urussanga SC.

\begin{tabular}{|c|c|c|c|c|c|c|}
\hline \multirow{2}{*}{ Mês } & \multirow{2}{*}{ Valores } & \multicolumn{5}{|c|}{ Período (dias) } \\
\hline & & 1 & 5 & 10 & 15 & 30 (mensal) \\
\hline \multirow{4}{*}{ janeiro } & Média $\left(\mathrm{mm} \mathrm{dia}^{-1}\right)$ & 3,94 & 3,92 & 3,92 & 3,91 & 3,92 \\
\hline & Desvio $\left(\mathrm{mm} \mathrm{dia}^{-1}\right)$ & 1,22 & 0,75 & 0,56 & 0,46 & 0,26 \\
\hline & $\mathrm{As}^{1}$ & 0,19 & 0,38 & 0,41 & 1,25 & 1,44 \\
\hline & C.V. ${ }^{2}(\%)$ & 30,96 & 19,13 & 14,29 & 11,76 & 6,63 \\
\hline \multirow{4}{*}{ fevereiro } & Média $\left(\mathrm{mm} \mathrm{dia}^{-1}\right)$ & 3,75 & 3,72 & 3,71 & 3,70 & 3,71 \\
\hline & Desvio $\left(\mathrm{mm} \mathrm{dia}^{-1}\right)$ & 1,21 & 0,76 & 0,61 & 0,50 & 0,42 \\
\hline & $\mathrm{As}^{1}$ & 0,28 & 0,46 & 0,79 & 0,71 & 0,60 \\
\hline & C.V. ${ }^{2}(\%)$ & 32,27 & 20,43 & 16,44 & 13,51 & 11,32 \\
\hline \multirow{4}{*}{ março } & Média $\left(\mathrm{mm} \mathrm{dia}^{-1}\right)$ & 3,23 & 3,22 & 3,21 & 3,20 & 3,21 \\
\hline & Desvio $\left(\mathrm{mm} \mathrm{dia}^{-1}\right)$ & 0,99 & 0,66 & 0,48 & 0,40 & 0,26 \\
\hline & $\mathrm{As}^{1}$ & 0,04 & 0,31 & 0,10 & 0,03 & 0,16 \\
\hline & C.V. ${ }^{2}(\%)$ & 30,65 & 20,49 & 14,91 & 12,57 & 7,98 \\
\hline \multirow{4}{*}{ abril } & Média $\left(\mathrm{mm} \mathrm{dia}^{-1}\right)$ & 2,42 & 2,40 & 2,40 & 2,39 & 2,39 \\
\hline & Desvio $\left(\mathrm{mm} \mathrm{dia}^{-1}\right)$ & 0,75 & 0,47 & 0,39 & 0,32 & 0,20 \\
\hline & $\mathrm{As}^{1}$ & 0,25 & 0,07 & 0,30 & 0,27 & 0,11 \\
\hline & C.V. ${ }^{2}(\%)$ & 30,99 & 19,58 & 16,25 & 13,39 & 8,37 \\
\hline \multirow{4}{*}{ maio } & Média $\left(\mathrm{mm} \mathrm{dia}^{-1}\right)$ & 1,71 & 1,69 & 1,69 & 1,68 & 1,68 \\
\hline & Desvio $\left(\mathrm{mm} \mathrm{dia}^{-1}\right)$ & 0,54 & 0,37 & 0,29 & 0,25 & 0,18 \\
\hline & $\mathrm{As}^{1}$ & 0,49 & 0,39 & 0,40 & 0,37 & 0,19 \\
\hline & C.V. ${ }^{2}(\%)$ & 31,58 & 21,89 & 17,16 & 14,88 & 10,71 \\
\hline \multirow{4}{*}{ junho } & Média $\left(\mathrm{mm} \mathrm{dia}^{-1}\right)$ & 1,37 & 1,34 & 1,33 & 1,32 & 1,31 \\
\hline & Desvio $\left(\mathrm{mm} \mathrm{dia}^{-1}\right)$ & 0,50 & 0,26 & 0,18 & 0,16 & 0,12 \\
\hline & $\mathrm{As}^{1}$ & 1,91 & 0,32 & 0,03 & 0,10 & 0,51 \\
\hline & C.V. ${ }^{2}(\%)$ & 36,50 & 19,40 & 13,53 & 12,12 & 9,16 \\
\hline \multirow{4}{*}{ julho } & Média $\left(\mathrm{mm} \mathrm{dia}^{-1}\right)$ & 1,47 & 1,44 & 1,42 & 1,42 & 1,40 \\
\hline & Desvio (mm dia $\left.{ }^{-1}\right)$ & 0,55 & 0,35 & 0,24 & 0,20 & 0,14 \\
\hline & $\mathrm{As}^{1}$ & 1,33 & 0,89 & 0,48 & 0,66 & $-0,26$ \\
\hline & C.V. ${ }^{2}(\%)$ & 37,41 & 24,31 & 16,90 & 14,08 & 10,00 \\
\hline \multirow{4}{*}{ agosto } & Média $\left(\mathrm{mm} \mathrm{dia}^{-1}\right)$ & 1,99 & 1,94 & 1,92 & 1,93 & 1,90 \\
\hline & Desvio $\left(\mathrm{mm} \mathrm{dia}^{-1}\right)$ & 0,78 & 0,48 & 0,35 & 0,29 & 0,18 \\
\hline & $\mathrm{As}^{1}$ & 0,96 & 0,53 & 0,15 & $-0,21$ & $-0,89$ \\
\hline & C.V. ${ }^{2}(\%)$ & 39,20 & 24,74 & 18,23 & 15,03 & 9,47 \\
\hline \multirow{4}{*}{ setembro } & Média $\left(\mathrm{mm} \mathrm{dia}^{-1}\right)$ & 2,49 & 2,45 & 2,42 & 2,44 & 2,43 \\
\hline & Desvio $\left(\mathrm{mm} \mathrm{dia}^{-1}\right)$ & 0,96 & 0,55 & 0,40 & 0,37 & 0,21 \\
\hline & $\mathrm{As}^{1}$ & 0,73 & 0,51 & 0,52 & 0,70 & $-0,17$ \\
\hline & C.V. ${ }^{2}(\%)$ & 38,55 & 22,45 & 16,53 & 15,16 & 8,64 \\
\hline \multirow{4}{*}{ outubro } & Média $\left(\mathrm{mm} \mathrm{dia}^{-1}\right)$ & 3,23 & 3,19 & 3,17 & 3,18 & 3,17 \\
\hline & Desvio $\left(\mathrm{mm} \mathrm{dia}^{-1}\right)$ & 1,21 & 0,76 & 0,58 & 0,47 & 0,33 \\
\hline & $\mathrm{As}^{1}$ & 0,44 & 0,63 & 0,59 & 0,31 & $-0,44$ \\
\hline & C.V. ${ }^{2}(\%)$ & 37,46 & 23,82 & 18,30 & 14,78 & 10,41 \\
\hline \multirow{4}{*}{ novembro } & Média $\left(\mathrm{mm} \mathrm{dia}^{-1}\right)$ & 3,85 & 3,82 & 3,81 & 3,80 & 3,80 \\
\hline & Desvio $\left(\mathrm{mm} \mathrm{dia}^{-1}\right)$ & 1,36 & 0,84 & 0,64 & 0,51 & 0,43 \\
\hline & $\mathrm{As}^{1}$ & 0,28 & 0,08 & $-0,26$ & $-0,68$ & $-0,44$ \\
\hline & C.V. ${ }^{2}(\%)$ & 35,32 & 22,04 & 16,75 & 13,45 & 11,23 \\
\hline \multirow{4}{*}{ dezembro } & Média $\left(\mathrm{mm} \mathrm{dia}^{-1}\right)$ & 4,04 & 4,00 & 4,00 & 4,00 & 4,00 \\
\hline & Desvio $\left(\mathrm{mm} \mathrm{dia}^{-1}\right)$ & 1,28 & 0,72 & 0,49 & 0,40 & 0,27 \\
\hline & $\mathrm{As}^{1}$ & 0,15 & 0,12 & 0,43 & 0,67 & 0,74 \\
\hline & C.V. ${ }^{2}(\%)$ & 31,68 & 18,01 & 12,13 & 10,02 & 6,63 \\
\hline
\end{tabular}

${ }^{1}$ As - coeficiente de assimetria ${ }^{2}$ C.V. - coeficiente de variação 
TABELA 2. Desvios máximos entre as frequiências observadas e as frequiências estimadas pela distribuição normal e distribuição Beta para valores de evapotranspiração de referência de Urussanga - SC, calculada em diversos intervalos.

\begin{tabular}{|c|c|c|c|c|c|c|c|c|c|c|}
\hline \multirow{3}{*}{ Mês } & \multicolumn{10}{|c|}{ Intervalo (dias) } \\
\hline & \multicolumn{2}{|c|}{1} & \multicolumn{2}{|c|}{5} & \multicolumn{2}{|c|}{10} & \multicolumn{2}{|c|}{15} & \multicolumn{2}{|c|}{30 (mensal) } \\
\hline & Normal & Beta & Normal & Beta & Normal & Beta & Normal & Beta & Normal & Beta \\
\hline jan. & $0,071^{*}$ & 0,040 & 0,067 & 0,059 & 0,090 & 0,048 & 0,113 & 0,087 & 0,113 & 0,093 \\
\hline fev. & $0,066^{*}$ & 0,042 & 0,049 & 0,046 & 0,103 & 0,066 & 0,110 & 0,107 & 0,098 & 0,099 \\
\hline mar & $0,070^{*}$ & 0,048 & 0,044 & 0,048 & 0,058 & 0,037 & 0,077 & 0,043 & 0,086 & 0,113 \\
\hline abr. & $0,061 *$ & 0,046 & 0,042 & 0,034 & 0,070 & 0,044 & 0,093 & 0,091 & 0,104 & 0,093 \\
\hline maio & 0,040 & 0,024 & 0,110 & 0,073 & 0,084 & 0,048 & 0,095 & 0,042 & 0,066 & 0,089 \\
\hline jun. & $0,084 *$ & 0,047 & 0,094 & 0,061 & 0,053 & 0,046 & 0,067 & 0,074 & 0,086 & 0,064 \\
\hline jul. & $0,084 *$ & 0,036 & 0,056 & 0,045 & 0,059 & 0,077 & 0,088 & 0,099 & 0,096 & 0,097 \\
\hline ago. & $0,073 *$ & 0,031 & 0,071 & 0,051 & 0,065 & 0,044 & 0,062 & 0,051 & 0,162 & 0,101 \\
\hline set. & $0,126^{*}$ & $0,066^{*}$ & 0,079 & 0,055 & 0,097 & 0,076 & 0,113 & 0,047 & 0,123 & 0,075 \\
\hline out. & $0,105^{*}$ & $0,058^{*}$ & 0,051 & 0,044 & $0,114 *$ & 0,107 & 0,080 & 0,041 & 0,088 & 0,071 \\
\hline nov. & $0,083^{*}$ & 0,051 & 0,052 & 0,036 & 0,088 & 0,035 & 0,114 & 0,079 & 0,102 & 0,079 \\
\hline dez. & $0,071^{*}$ & 0,055 & 0,064 & 0,044 & 0,088 & 0,082 & 0,082 & 0,084 & 0,111 & 0,091 \\
\hline
\end{tabular}

* significativo a $5 \%$.

Na Figura 1, estão representados os valores de evapotranspiração de referência calculados em intervalos diários com diferentes níveis de probabilidade para os diversos meses do ano. Nas Figuras 2 e 3, estão representados os valores de evapotranspiração de referência com diferentes níveis de probabilidade, calculadas com diferentes intervalos de tempo para janeiro e julho, respectivamente.

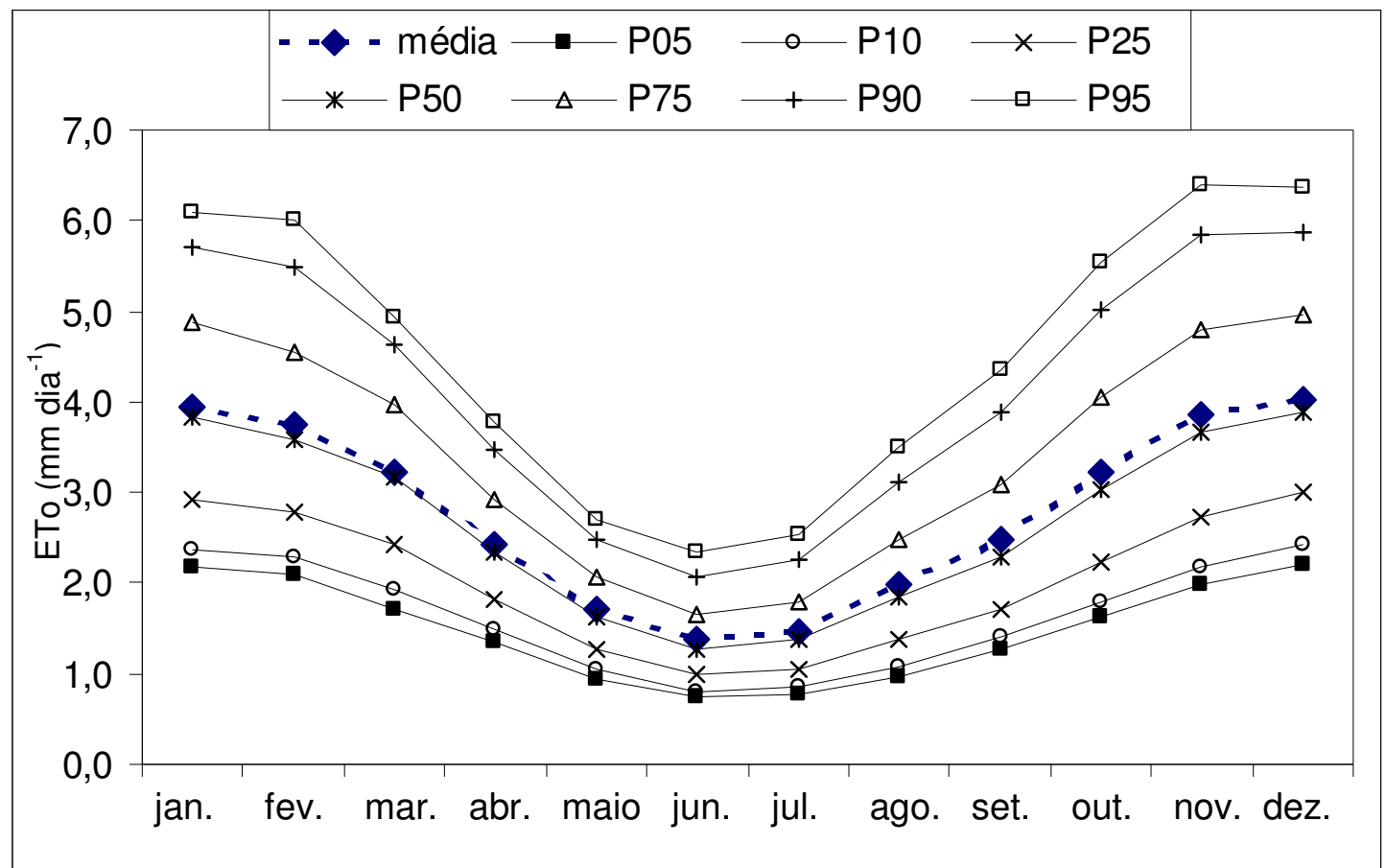

FIGURA 1. Evapotranspiração de referência calculada com dados diários com probabilidade de 5\% (P5), 10\% (P10), 25\% (P25), 50\% (P50), 75\% (P75), 90\% (P90) e 95\% (P95) para Urussanga - SC.

Nos projetos de irrigação, a escolha do nível de probabilidade depende das condições econômicas e do risco admissível. DOOREMBOS \& PRUITT (1984) recomendam valores entre 75 e 80\%; nas condições de irrigação suplementar típicas da região Sul do Brasil, dificilmente se recomendam valores superiores a $80 \%$ de probabilidade. O valor da evapotranspiração de 
referência com $75 \%$ de freqüência tem sido recomendado para o dimensionamento de projetos de irrigação. No entanto, como o período de máxima demanda hídrica não se restringe a um dia, devese considerar a duração desse período. JENSEN (1974) recomenda um período de duas a três semanas.

Analisando as Figuras 2 e 3, observa-se que, para períodos acima de dez dias, o valor da evapotranspiração de referência com $75 \%$ de probabilidade tende a manter o mesmo valor, aumentando significativamente para períodos de cinco dias ou menores. Comparando os valores de evapotranspiração com $75 \%$ de probabilidade, calculada em intervalos diários, pêntados, decendiais e quinzenais, com o valor calculado para intervalos mensais, observam-se diferenças médias de 17,0; 8,4; 4,8 e 2,9\%, respectivamente. Dessa forma, pode-se concluir que adotar o valor de evapotranspiração com $75 \%$, calculada em intervalos diários ou de pêntadas, pode superestimar o valor da demanda hídrica se o período crítico é superior a dez dias. Por outro lado, adotar o valor da evapotranspiração calculada com base mensal pode subestimar o valor se o período crítico for menor que dez dias.

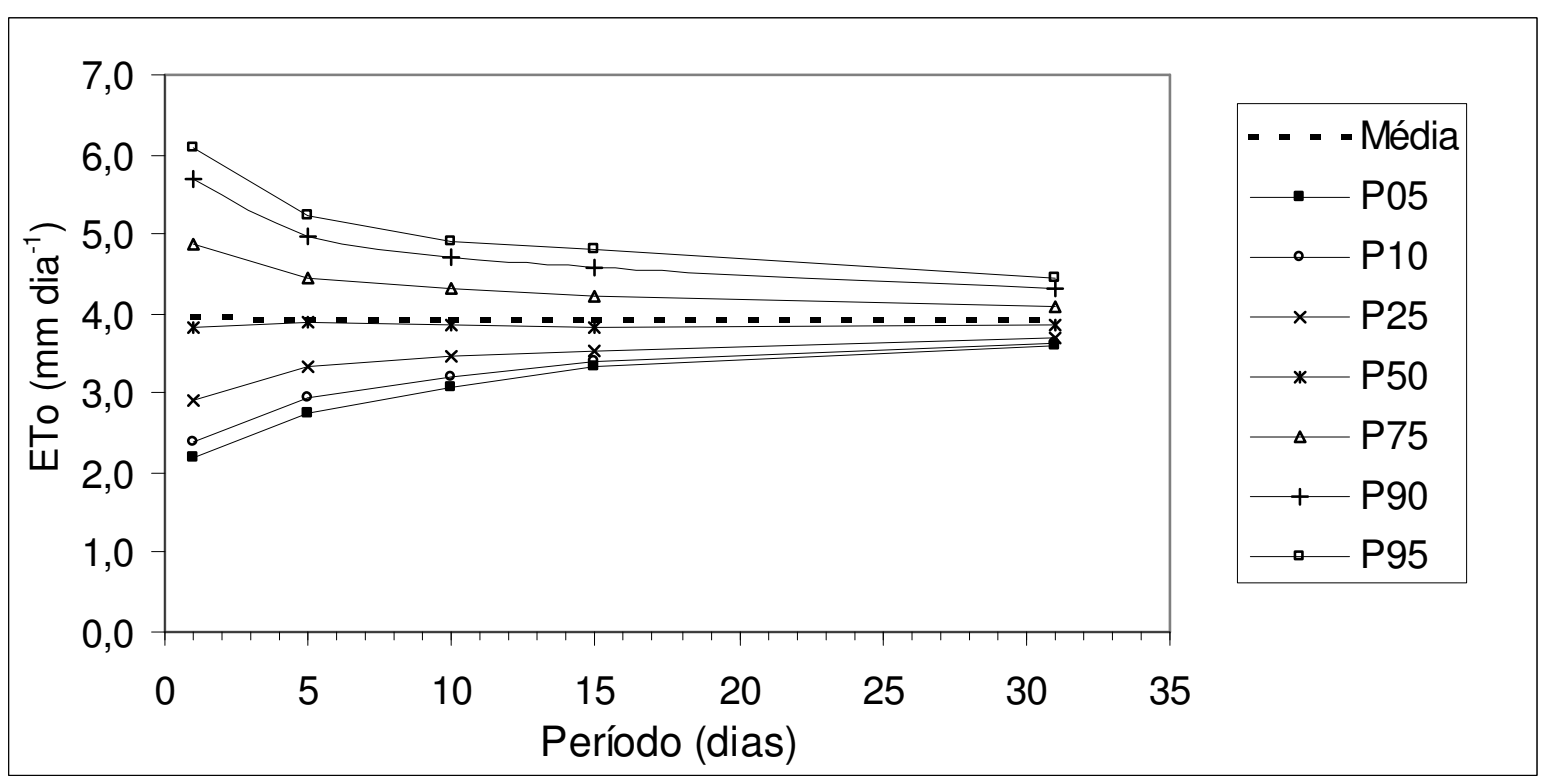

FIGURA 2. Variação da evapotranspiração de referência calculada com dados de diferentes períodos para janeiro, com probabilidade de 5\% ( P5), 10\% (P10), 25\% (P25), 50\% (P50), 75\% (P75), 90\% (P90) e 95\% (P95), para Urussanga - SC.

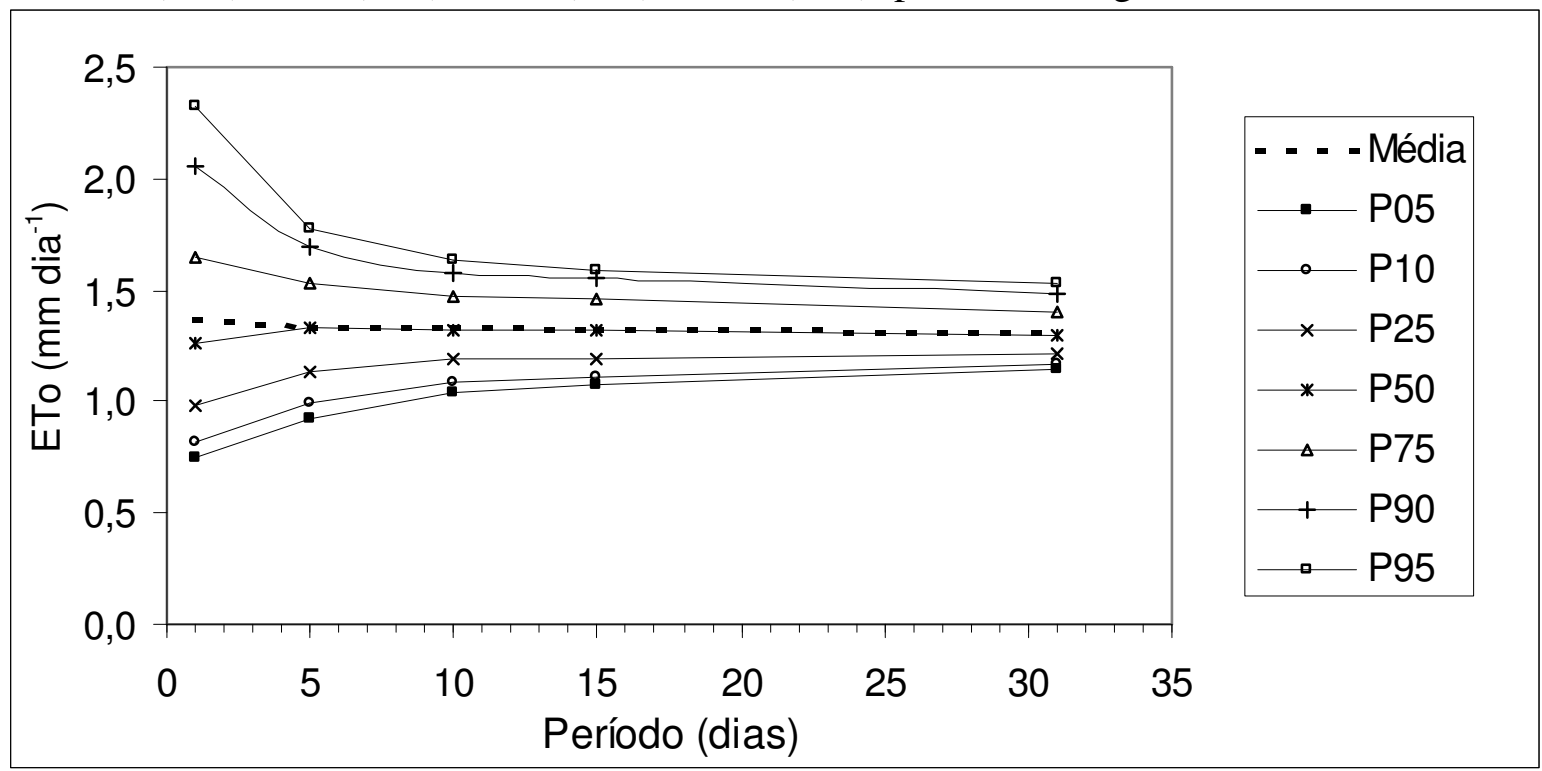

FIGURA 3. Variação da evapotranspiração de referência calculada com dados de diferentes períodos para julho, com probabilidade de $5 \%$ (P5), 10\% (P10), 25\% (P25), 50\% (P50), 75\% (P75), 90\% (P90) e 95\% (P95), para Urussanga - SC. 
Com esses dados, observa-se que a escolha do valor da evapotranspiração de referência a ser utilizado no projeto de irrigação deve ser realizada de acordo com a duração do período de máxima demanda e do risco associado ao projeto. Observa-se também que, embora a evapotranspiração de referência não varie tanto quanto a precipitação, as variações observadas nos valores justificam o uso de teorias de probabilidades para a obtenção dos valores de projeto em vez de utilizar os dados médios.

\section{CONCLUSÕES}

Os valores de evapotranspiração de referência média não são diferentes estatisticamente ( $>>0,10)$, calculados com intervalos de um, cinco, dez, 15 dias ou mensais.

Para intervalos de um dia, obteve-se coeficiente de variação entre 30 e $40 \%$, e para intervalos mensais, o coeficiente de variação situou-se entre 6 e $12 \%$.

A distribuição Beta ajustou-se melhor aos dados de evapotranspiração de referência do que a distribuição normal. A distribuição normal foi rejeitada para os valores de evapotranspiração de referência calculados em intervalos diários.

A evapotranspiração calculada com $75 \%$ de probabilidade para intervalo de um, cinco, dez e 15 dias é, em média, 17,0; 8,4; 4,8 e 2,9\% superior à evapotranspiração de $75 \%$ de probabilidade, calculada com intervalos mensais.

\section{REFERENCIAS}

ALLEN, R.G.; PEREIRA, L.S.; RAES, D.; SMITH, M. Crop evapotranspiration: guidelines for computing crop water requirements. Rome: FAO, 1998. (Irrigation and Drainage, 56)

ARRUDA, F.B.; BARROSO, L.F.S. Estimativa do uso de água para fins de projetos de irrigação em função da evaporação de tanque, em Ribeirão Preto. Bragantia, Campinas, v.43, n.2, p.677-82, 1984.

ASSIS, F.N.; ARRUDA, H.V.; PEREIRA, A.R. Aplicações de estatística à climatologia: teoria e prática. Pelotas: Universitária/UFPEL, 1996. 191 p.

DOOREMBOS, J.; PRUITT, W.O. Guidelines for predicting crop water requirements. Rome: FAO, 1984. 144 p.

JENSEN, M.E. Consumptive use of water and irrigation water requirements. New York: ASCE, 1974. $215 \mathrm{p}$.

JENSEN, M.E.; BURMAN, R.D.; ALLEN, R.G. Evapotranspiration and irrigation water requirements. New York: American Society of Civil Engineers, 1990. 329 p. (ASCE Manual and Report on Engineering Practice, 70).

KITE, G.H. Frequency and risk analyses in hydrology. Fort Collins: Water Resources Publications, 1978. 224 p.

LIMA, A.S.F.F. Comparação de métodos de estimativa de evapotranspiração potencial para o município de Ribeirão Preto - SP. 1991. 169 f. Dissertação (Mestrado em Irrigação e Drenagem) Escola Superior de Agricultura "Luiz de Queiroz", Universidade de São Paulo, Piracicaba,1991.

SAAD, J.C.C.; SCALOPPI, E.J. Freqüência de distribuição de evapotranspiração de referência para o dimensionamento de sistemas de irrigação. In: CONGRESSO NACIONAL DE IRRIGAÇÃO E DRENAGEM, 9., 1988, Florianópolis. Anais... Florianópolis: ABID, 1988. v.2, p.1037-52.

SAAD, J.C.C. Estudo das distribuições de freqüências da evapotranspiração de referência e da precipitação pluvial para fins de dimensionamento de sistemas de irrigação. 1990. $124 \mathrm{f}$. Dissertação (Mestrado em Irrigação e Drenagem) - Escola Superior de Agricultura "Luiz de Queiroz”, Universidade de São Paulo, Piracicaba, 1990.

SMITH, M. Report on the expert consultations on revision of FAO methodologies for crop water requirements. Rome: FAO, 1991. 45 p. 